\title{
Buscando condiciones de adecuación para la reconstrucción de teorías*
}

\author{
Looking for Conditions of Adequacy for the Reconstruction of Theories
}

Luis Miguel Peris-Viñé $e^{\dagger}$

\begin{abstract}
Resumen
En filosofía especial de la ciencia se hace necesario disponer de criterios de justificación de las reconstrucciones propuestas. Pero los filósofos se han ocupado mucho de la justificación de las teorías y poco de la justificación de las reconstrucciones de las teorías. Para precisar los criterios de justificación de las reconstrucciones propongo una analogía con el proceso de justificación de las gramáticas en el ámbito de la lingüistica, equiparando la estratificación lenguajegramática de lenguaje-teoría general de este proceso con la estratificación correspondiente teoría-reconstrucción de la teoríametateoría del proceso de justificación de las reconstrucciones. El desarrollo de esta analogía deberá afrontar el establecimiento de condiciones externas y de condiciones internas de adecuación de las reconstrucciones. Defenderé un principio de dependencia estructural de la ontología y la epistemología. Mediante este principio se analizará aspectos básicos de teorías lingüisticas particulares desde el punto de vista del mentalismo y del platonismo.
\end{abstract}

Palabras clave: reconstrucción de teorías - filosofía de la lingüistica - mentalismo - Chomsky

\begin{abstract}
In philosophy of special sciences is necessary to have criteria of justification of the proposed reconstructions. But the philosophers have dedicated a lot of time to the justification of theories and little to the justification of the reconstructions of theories. I propose, for specifying the criteria of justification of reconstructions, an analogy with the process of justification of grammars in the environment of linguistics, and I compare the language-grammar of language-general theory stratification of that process with the theory-reconstruction of the theory-metatheory stratification corresponding to the process of justification of reconstructions. The development of this analogy will face the establishment of external conditions and of internal conditions of adequacy of reconstructions. I will defend a principle of structural dependence of ontology and epistemology. By means of this principle it will be analyzed basic aspects of particular linguistic theories from the point of view of mentalism and of Platonism.
\end{abstract}

Keywords: reconstruction of theories - philosophy of linguistics - mentalism - Chomsky

\footnotetext{
* Recibido: 13 de marzo de 2013. Aceptado con revisiones: 10 de julio de 2013.

† Universidad de Granada, España. Para contactar al autor, por favor, escribir a: perisv@ugr.es. Metatheoria 6(1)(2015): 39-45. ISSN 1853-2322. eISSN 1853-2330.

(C) Editorial de la Universidad Nacional de Tres de Febrero. Publicado en la República Argentina.
} 
Mi interés por la metateoría de la ciencia y en particular por la metateoría estructural y sus potencialidades procede de mis problemas personales. Problemas personales, aunque relativos a la naturaleza de los objetos que estudia una ciencia particular, la lingüística. Y concretamente a los aspectos ontológico, epistemológico y estructural de su naturaleza. Tales problemas radican en las dificultades existentes para identificar los perfiles de esos aspectos de su naturaleza.

Hay un tipo de cuestión filosófica general que consiste en preguntar qué hay. Con frecuencia los filósofos de la ciencia insisten en considerar que una metateoría como la metateoría estructural, al llevar a cabo reconstrucciones de teorías científicas, no necesita comprometerse con una respuesta a esa cuestión filosófica general, no presupone ningún compromiso ontológico determinado. Ahora bien, hay otro tipo de cuestión filosófica, particular, menor quizás para algunos, que consiste en preguntar qué hay según la teoría T. En relación a este tipo de cuestiones no deberíamos olvidar que una metateoría como la metateoría estructural puede servirnos para identificar los compromisos ontológicos de las teorías. Mi presunción es que la cuestión ontológica general debe abordarse desde la respuesta a las cuestiones ontológicas particulares.

La idea básica que aquí quiero proponer, y desarrollar parcialmente, respecto de la lingüística es que si dispusiéramos de condiciones de adecuación para la reconstrucción de nuestras teorías podríamos identificar adecuadamente el tipo de objetos de las teorías lingüísticas, sus compromisos ontológicos y podríamos evaluar la corrección de las propuestas explicativas de los lingüistas.

A lo largo del siglo XX se han producido aportaciones muy contundentes, determinantes, de la configuración de las teorías lingüísticas. Las más importantes de tales aportaciones han estado referidas al tipo de objetos a los que la lingüística debe o puede dirigirse. Las concepciones ontológicas más extendidas en la lingüística del siglo $\mathrm{XX}$ son herederas de las concepciones mantenidas en las discusiones medievales sobre los universales: nominalismo (no hay universales, y los términos que los nombran se refieren a semejanzas sensibles entre particulares), conceptualismo (los universales son mentales) y realismo (los universales son reales, aunque ni físicos ni mentales). Las aportaciones de los lingüistas a este respecto divergen en gran medida. Incluso, si nos fijamos con suficiente atención, podremos apreciar algo sorprendente, que un mismo lingüista defienda concepciones contrarias al respecto a lo largo de su obra aunque el mismo no aprecie las diferencias entre ellas. Es el caso de Leonard Bloomfield, que en Bloomfield (1914) se muestra mentalista mientras que en Bloomfield (1933) se muestra nominalista, fisicalista y antimentalista. Y también es el caso de Noam Chomsky, que en Chomsky (1955) se muestra antimentalista, partidario de Bloomfield (1933), quizás realista, mientras que en Chomsky (1965) se muestra conceptualista, mentalista naturalista y contrario a Bloomfield (1933).

Mi preocupación por la justificación de las reconstrucciones de teorías parte de considerar que tanto la construcción de teorías científicas como la reconstrucción de teorías científicas son empresas cognoscitivas. Tales empresas son intencionales, en el sentido de que los agentes cognoscitivos que intervienen en ellas pretenden obtener conocimiento sobre cierto asunto. La racionalidad de este tipo de pretensiones exige disponer de condiciones de adecuación. En nuestro caso, de condiciones de adecuación bien de las teorías bien de las reconstrucciones de las teorías. En el diseño de tales condiciones de adecuación ha de tenerse en cuenta que los asuntos de las teorías y los de las reconstrucciones son parcialmente externos, de naturaleza independiente de los agentes y de sus intenciones. Sabemos que las condiciones de adecuación de las teorías científicas han sido muy estudiadas, no así las condiciones de adecuación de las reconstrucciones de las teorías. Algunos lingüistas han reflexionado sobre las condiciones de adecuación de sus teorías. Consideraré aquí alguna de tales reflexiones para abordar ciertas cuestiones sobre la adecuación de las reconstrucciones. Me fijaré en el punto de vista de Chomsky (1955) y de Chomsky (1965).

Según Chomsky (1955), la tarea de la lingüística descriptiva consta de tres áreas: la construcción de gramáticas para lenguajes particulares, la formulación de una teoría general de la estructura lingüística, de la cual las distintas gramáticas sean ejemplificaciones y la justificación y validación de los resultados. Estas tres áreas están relacionadas. Así, una gramática particular "purports to show the 'structure' of 
the language" (Chomsky 1955, p. 80). "Ultimately the purpose of a grammar is to provide a specification and description of the grammatical utterances of the language" (Chomsky 1955, p. 167). "A sentence token can be represented as a sequence of phonemes; but it can also be represented as a sequence of morphemes, words, and phrases. Thus each sentence token will have associated with it a whole set of representations, each representation being its 'spelling' in terms of elements of one linguistic level” (Chomsky 1955, p. 99). Estos objetivos de una gramática particular son acometidos mediante su organización en varios niveles para la representación de las oraciones de una lengua: "a grammar of a language must state the structure of each grammatical utterance of the language on each linguistic level" (Chomsky 1955, p. 107). Esta representación es tal que constituye una especificación de las oraciones gramaticales de la lengua. Por su parte, la teoría lingüística general "becomes the theory in which these systems of representation are constructed and studied in an abstract manner, and the relations between them explicitly characterized" (Chomsky 1955, p. 99), "the abstract theory in which the basic concepts of grammar are developed, and by means of which each proposed grammar can be evaluated" (Chomsky 1955, p. 63). De modo que "every grammar must be compatible with the theory in the sense that the elements set up in the grammar must exhibit the general properties required by the theory" (Chomsky 1955, p. 79). Las gramáticas particulares deben ser modelos de la teoría general, deben ejemplificar la teoría general (ver Chomsky 1955, p. 80).

Además, existen modos prácticos de relacionarse las gramáticas con la teoría general. Por ejemplo, la teoría lingüística podría proporcionar un método de evaluación de la gramáticas propuestas, "so that, given two proposed grammars, there would be a practical and mechanical way for determinig which is the better of the two" Chomsky $(1955$, p. 79). Una relación más fuerte que ésta sería una por la que la teoría lingüística proporcionaría un método de decisión que establecería si determinada gramática es o no la mejor gramática. Y finalmente la relación más fuerte de todas sería una por la que la teoría lingüística proporcionaría un método de descubrimiento de la gramática para una lengua dado un corpus. Los siguientes gráficos representan estos modos de relación:
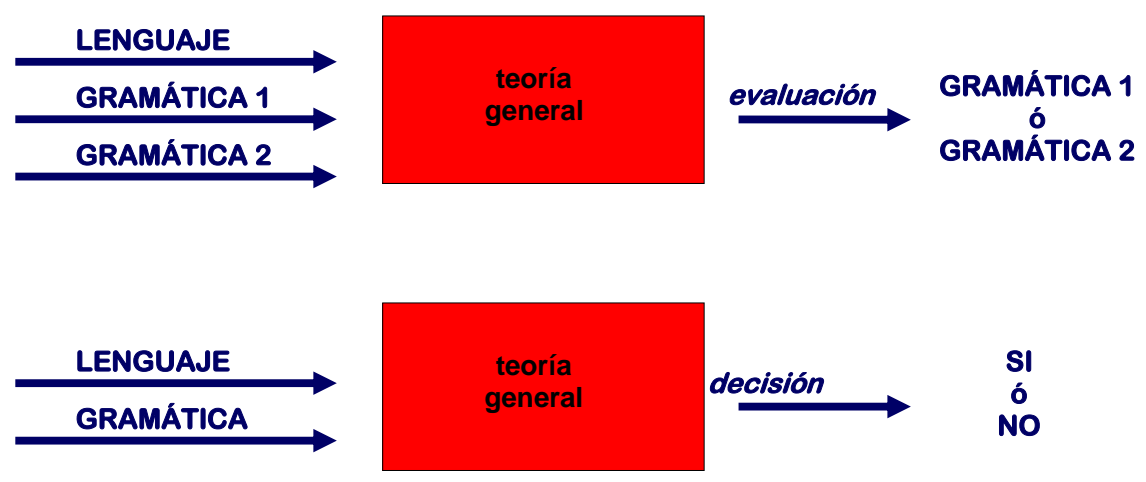

SI
ó
NO

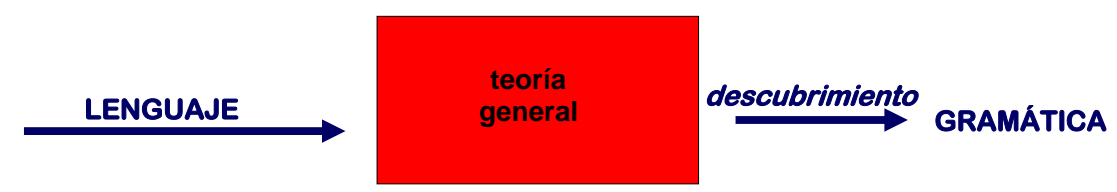

Según Chomsky (1955, p. 79), a lo más que podemos aspirar es a que la teoría lingüística ofrezca un método de evaluación de gramáticas. Para Chomsky, la relación más débil de las tres, "el más débil de esos requisitos es todavía lo bastante fuerte como para garantizar la significatividad de una teoría que lo satisface. Hay pocas áreas de la ciencia en las que sería posible considerar seriamente la posibilidad de desarrollar un método general mecánico y práctico para seleccionar entre varias teorías, compatibles todas y cada una de ellas con los datos de que se dispone" (Chomsky 1957, p. 71). 
Llegados a este punto y para ayudar a precisar las aspiraciones respecto de la adecuación de las reconstrucciones que vengo considerando propongo partir de las siguientes analogías entre lingüistica y filosofía de la ciencia:
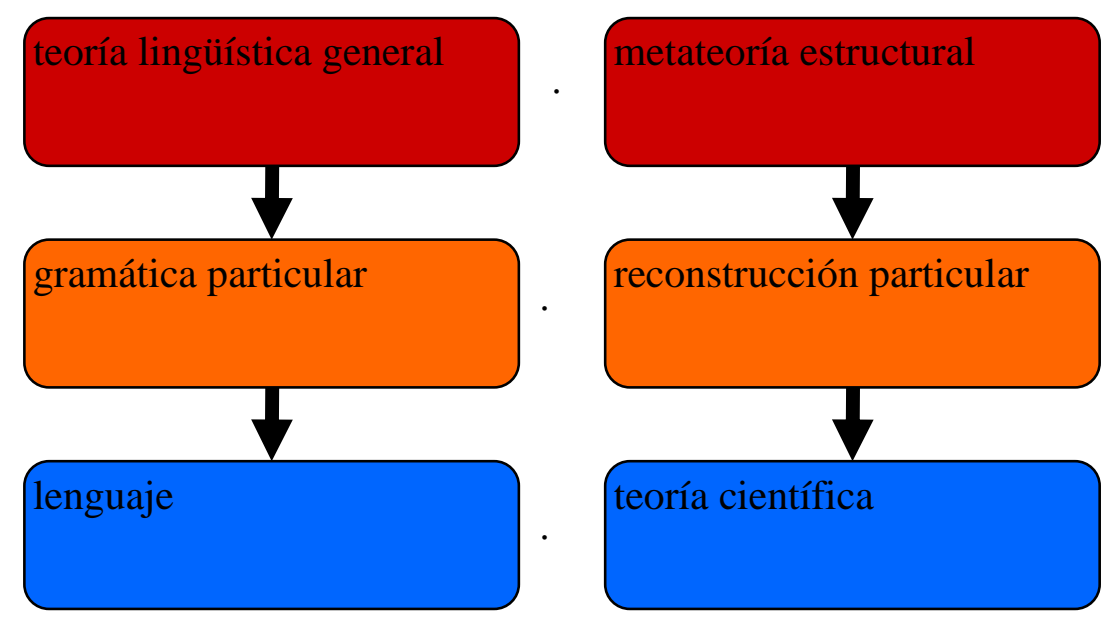

Es decir, si una gramática particular tiene por objeto un lenguaje natural y la teoría lingüística general es una reflexión metateórica sobre las gramáticas particulares, de modo semejante la reconstrucción de una teoría tiene por objeto dicha teoría y la metateoría estructural es una reflexión metateórica sobre las reconstrucciones particulares. Establecidas estas analogías, las relaciones posibles entre la metateoría estructural, las reconstrucciones particulares y las teorías serían las siguientes:

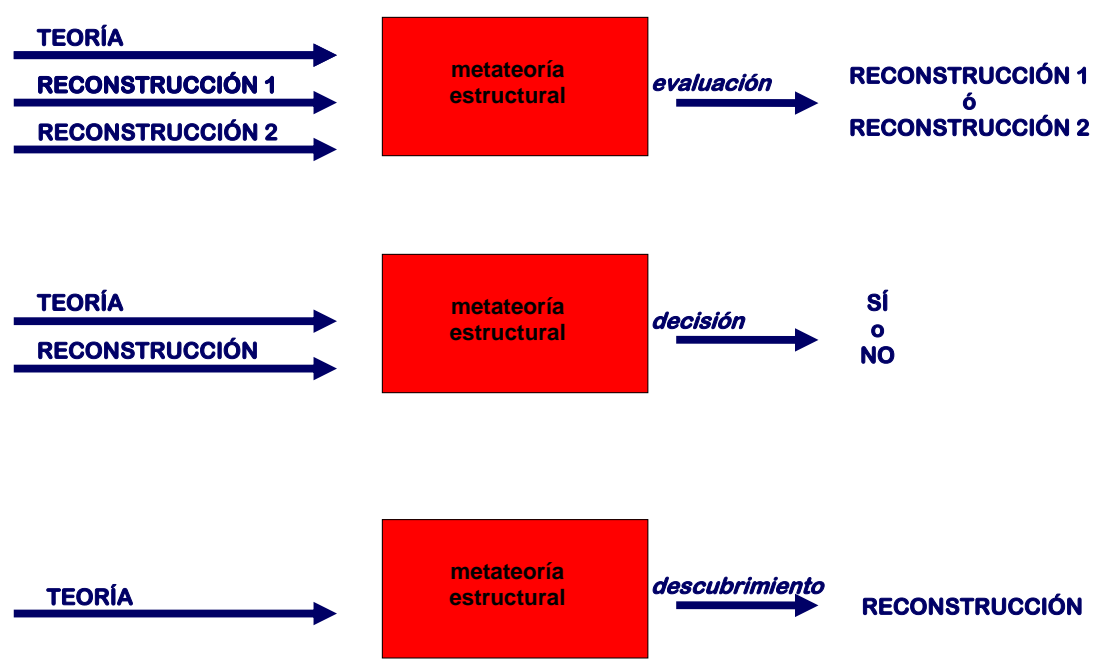

Así, si en la ciencia en general y en la lingüistica en particular sólo podemos aspirar a métodos de evaluación (nunca de decisión o descubrimiento) en el ámbito de la reconstrucción de teorías sucede lo mismo, sólo podemos aspirar a métodos o condiciones de evaluación.

No obstante lo dicho conviene hacer un par de advertencias. Hay que tener en cuenta que del mismo modo que el lingüista no tiene acceso al lenguaje en su totalidad sino a corpus seleccionados y parcialmente informativos y que pueden ser más o menos representativos, también al filósofo le sucede lo mismo, pues éste no tiene acceso a la teoría en su totalidad sino a fragmentos de la misma y a aplicaciones seleccionados y parcialmente informativos que pueden ser más o menos representativos. Por otro lado quienes consideren que la elaboración de reconstrucciones particulares no es un método privilegiado de conocimiento de las teorías científicas frente a otros procedimientos, tales como el "análisis fino y certero" o la "lectura atenta" y que por tanto no asegura una mayor tasa de éxito que 
estos otros procedimientos deberían igualmente considerar que la elaboración de gramáticas (o de teorías científicas en general) no es un método privilegiado para conocer el lenguaje (o el objeto de la teoría científica de la que se trate) más allá de la "consideración atenta" de su objeto. No existen procedimientos que aseguren el éxito cognoscitivo, ni en la ciencia, ni en la lingüística, ni en la metateoría filosófica. Lo que sí tenemos es la posibilidad de evaluar los mejores métodos disponibles. Las teorías científicas (y entre ellas las gramáticas particulares de los lenguajes naturales) y las reconstrucciones particulares de las mismas constituyen los mejores procedimientos disponibles para sus respectivas tareas cognoscitivas.

Pero ¿cómo puede la teoría lingüistica general proporcionar un método de evaluación para las gramáticas particulares que permita elegir entre dos gramáticas propuestas? Es decir, ¿cómo justificar una gramática?, ¿qué condiciones debe cumplir? La manera de abordar el tema de la justificación de las gramáticas depende claramente de la concepción que se tenga de lo que es una gramática. En la corriente generativa en lingüística siempre se ha concebido una gramática como una teoría científica cuyo objeto de estudio es independiente de la intención de los agentes. Esto hace que la justificación de una gramática deba descansar, en parte, en el cumplimiento de ciertas condiciones externas de adecuación que indiquen cómo debe relacionarse una gramática con los datos relativos a su objeto de estudio. No hay acuerdo entre los lingüistas sobre en qué se cifran esas condiciones externas (intuición de los usuarios del lenguaje, juicios de los usuarios, conocimiento, uso, etc.). Pero, en cualquier caso, las condiciones externas no son suficientes para evaluar las gramáticas. Una gramática ha de satisfacer además condiciones internas de adecuación, lo cual significa que una gramática debe ser compatible o conformar con la teoría lingüística general. Para Chomsky, "the problem of justification and that of constructing a general theory of linguistic structure are, in part at least, essentially the same" (Chomsky 1955, p. 85). Ahora bien, a partir de los primeros años sesenta, trascurridos ya cinco años del inicio de la corriente generativa, Chomsky introduce una consideración nueva, según la cual la teoría lingüística general es una teoría del proceso de desarrollo de la lengua, concretamente de la base o precondición que el aprendiz posee y que permite y guía su aprendizaje/desarrollo. Tanto es así que, ahora, para Chomsky, el "problema de la justificación interna -de la adecuación explicativa- es, en esencia, el problema de construir una teoría de la adquisición del lenguaje, un dar cuenta de las habilidades innatas específicas que hacen posible este logro" (Chomsky 1965, p. 27).

Aquí, para plantear nuestra analogía entre gramáticas particulares y reconstrucciones particulares no necesitamos entrar en la discusión sobre el contenido de las condiciones que deberían exigirse, sólo interesa señalar que debería indagarse la posibilidad de formular tales condiciones de adecuación externas y condiciones de adecuación internas para las reconstrucciones particulares de teorías científicas y también señalar de dónde provendrían tales condiciones. Si del lenguaje provienen las condiciones externas de las gramáticas y de la teoría lingüística general provienen sus condiciones externas, indaguemos las opciones que existen de elaborar condiciones externas de las reconstrucciones que provengan de las teorías a reconstruir y condiciones internas que provengan de una metateoría como la estructural. Si algunos lingüistas consideran que para que estas conexiones tengan sentido y den lugar a resultados científicamente interesantes la teoría lingüística general ha de concebirse y elaborarse como una teoría del proceso natural de desarrollo de lenguaje, indaguemos la posibilidad de exigir que una metateoría como la estructural se conciba y se elabore como una teoría del proceso natural de desarrollo de las teorías científicas. En este caso, si los lingüistas consideran que para elaborar una buena gramática han de atender necesariamente a las experiencias lingüísticas y a la dotación biológica innata de los hablantes que permiten que surja el lenguaje, quizás, igualmente, los filósofos consideren que para elaborar una buena reconstrucción de una teoría científica se ha de atender necesariamente a procesos de formación teórica y axiológica, a actos de observación e investigación, etc., de los científicos pero también a la dotación biológica, en definitiva a todo aquello que permite que surjan las teorias científicas. De este modo queda preparado un tablero amplio de posibilidades en el cada cual puede situarse. En el siguiente gráfico quedan reflejadas las conexiones que articulan ese tablero de posibilidades: 


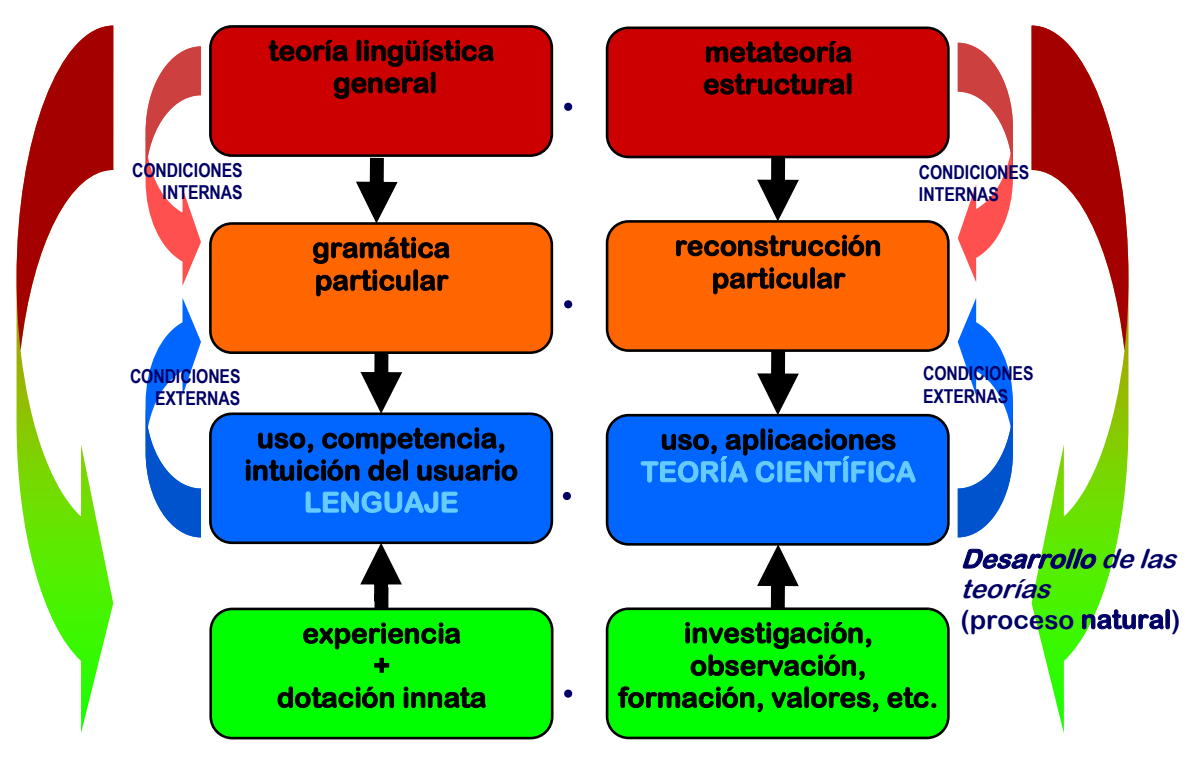

Considero que estas analogías entre gramáticas particulares y reconstrucciones particulares pueden ayudar a clarificar la tarea del filósofo pero también la del lingüista.

Retomemos ahora las disputas ontológicas de los lingüistas sobre la naturaleza de su objeto, el lenguaje, y transitemos desde una de las columnas del anterior gráfico a la otra. Como señalé al inicio, si dispusiéramos de condiciones de adecuación para las reconstrucciones de nuestras teorías lingüísticas podríamos identificar el tipo de objeto al que se dirigen, y contribuir a resolver, por ejemplo, la disputa entre nominalismo, conceptualismo y realismo respecto del lenguaje. Esta propuesta la podemos formular a modo de principio. Un principio denominado principio de dependencia estructural de la ontología y la epistemología, y según el cual aspectos cruciales del estatuto ontológico y epistemológico de las teorías lingüísticas dependen de la estructura de éstas. Cuáles son esos aspectos dependientes y en qué medida dependen de la estructura de las teorías será algo que habrá que averiguar mediante la reconstrucción continuada de teorías particulares, y especialmente de sus aplicaciones. Entre tales aspectos están los relativos al tipo de objetos, a la naturaleza de las nociones y a las relaciones entre teorias lingüísticas. Mi posición es que estos aspectos se manifiestan en la estructura de las teorías, por lo que la reconstrucción de la estructura de las teorías lingüísticas es un instrumento adecuado para dirimir ciertas discusiones sobre los mismos.

Por ejemplo la cuestión de si los objetos de cierta teoría lingüística son naturales o son abstractos puede responderse mediante la reconstrucción de la teoría afectada. Y es que sabemos que son los axiomas de interpretación de una teoría los que indican cuáles son los objetos de los que se ocupa esa teoría. De esos axiomas, los referidos al dominio básico podrán indicar, por ejemplo, si los objetos de la gramática son estados mentales, oraciones o configuraciones neurológicas; además, los referidos a las nociones relacionales indicarán el tipo de propiedades que esas nociones pretenden expresar del tipo de objetos ya señalado. Pues bien, si acometemos la tarea de reconstruir las teorías en las que nociones tales como estructura profunda, representación fonética, forma lógica, etc. aparecen, el resultado que obtengamos deberá indicarnos el tipo de objetos y el tipo de valores asignados por esas nociones. El resultado se mostrará al identificar y reconstruir los procedimientos de determinación de los valores de tales nociones. Algunas de las reconstrucciones de las que disponemos indican que los objetos son oraciones y que los valores son representaciones estructurales de esas oraciones (ver Peris-Viñé 1996, 2002, 2012). Aunque, claro, el procedimiento está abierto para argumentar en sentidos contrarios.

Otro ámbito de aspectos en los que el principio de dependencia estructural parece vigente es en el de la vinculación entre teorías, o partes de teorías. Por ejemplo, los defensores del mentalismo naturalizado establecen una condición para que la asignación de representaciones estructurales a las oraciones, efectuada por la gramática y fruto final, según ellos, de los principios generales que rigen el sistema 
computacional integrado en la facultad del lenguaje, pueda ser interpretada como una asignación de una propiedad psicológica a un objeto mental. Se trata de una condición sobre las relaciones interteóricas que deben darse entre las ramas de la ciencia afectadas. La condición es que tenga lugar un proceso de unificación de la lingüística mentalista con la neurología computacional. En ocasiones parece que ese proceso se concibe como un caso de reducción, aunque se evite este término, por el cual, nos daremos cuenta que, en realidad "los enunciados de la gramática son enunciados de la teoría de la mente sobre la lengua-I, y por lo tanto enunciados sobre estructuras del cerebro formulados en un cierto nivel de abstracción realizada sobre mecanismos" (Chomsky 1986, p. 38). Es evidente que para dirimir este asunto, que es de naturaleza ontológica, hemos de reconstruir previamente las relaciones estructurales de las teorías implicadas.

Otro ámbito de aplicación del principio de dependencia estructural lo constituyen las investigaciones que comparan teorías lingüisticas pertenecientes a distintas corrientes o tradiciones, bien se presenten tales teorías lingüísticas como competidoras alternativas o bien se presenten como versiones equivalentes.

Finalizaré con una recomendación, que se sigue de todo lo dicho. Precisemos condiciones de adecuación de las reconstrucciones estructurales a fin de identificar los compromisos ontológicos de las teorías particulares y, así, quizás, poder abordar la cuestión ontológica general.

Bibliografía

Bloomfield, L. (1933), Language, New York: Holt, Rinehart \& Winston.

Chomsky, N. (1955), The Logical Structure of Linguistic Theory, New York: Plenum, 1975.

Chomsky, N. (1957), Syntactic Structures, The Hague: Mouton. (Versión castellana de Carlos Peregrín Otero: Estructuras sintácticas, México: Siglo XXI, 1974.)

Chomsky, N. (1965), Aspects of the Theory of Syntax, Cambridge, MA: The MIT Press. (Versión castellana: Aspectos de la teoría de la sintaxis, Madrid: Aguilar, 1970.)

Chomsky, N. (1986), Knowledge of Language: Its Nature, Origins and Use, New York: Praeger Publishers. (Versión castellana: El conocimiento del lenguaje, Madrid: Alianza, 1989.)

Peris-Viñé, L. M. (1996), “Caracterización de las nociones básicas de la Gramática de Chomsky”, Ágora 15(2): 105-124.

Peris-Viñé, L. M. (2002), "Relaciones interteóricas en la lingüística actual”, en Díez, J. A. y P. Lorenzano (eds.), Desarrollos actuales de la metateoría estructuralista, Bernal: Universidad Nacional de Quilmes/Universidad Autónoma de Zacatecas/Universidad Rovira i Virgili, pp. 333-362.

Peris-Viñé, L.M. (2012), “Estructura parcial de la gramática estándar del castellano”, en Peris-Viñé, L. M. (ed.), Filosofía de la Ciencia en Iberoamérica: Metateoría estructural, Madrid: Tecnos, pp. 225-258. 